\title{
POSTULATES FOR BOOLEAN ALGEBRA IN TERMS OF TERNARY REJECTION*
}

\author{
BY ALBERT WHITEMAN $\dagger$
}

1. Introduction. The operation of ternary rejection $\ddagger$ in Boolean algebra is the operation ( ) given by $(a b c)=a^{\prime} b^{\prime}+b^{\prime} c^{\prime}+c^{\prime} a^{\prime}$. In this paper, I shall present a set of postulates for Boolean algebras in which ternary rejection is taken as the only primitive idea, besides that of class. As a result, all the special Boolean elements are introduced with an elegance not possible in any other set known to the author. Thus, the negative of an element is defined in terms of the primitive ideas, and then any two contradictory elements are chosen to represent the zero element and the universe element of the resulting Boolean algebra.

We prove the sufficiency of the new postulates for Boolean algebra by deriving from them the well known WhiteheadHuntington set; $\S$ the proof of necessariness consists in the converse derivation. Finally, we establish the consistency and independence of the postulates by furnishing proof-systems of the usual type.

2. The New Postulates. The new postulates have as undefined ideas a class $K$ and a ternary operation ( ). The postulates are the propositions $A_{1}-A_{5}$ below. In Postulates $A_{3}-A_{5}$ the condition if the elements involved and their indicated combinations belong to $K$ is to be understood.

Postulate $\mathrm{A}_{1} . K$ contains at least two distinct elements.

Postulate $\mathrm{A}_{2}$. If $a, b, c$ are elements of $K,(a b c)$ is an element of $K$.

* Presented to the Society, December 31, 1936.

$\dagger$ Harrison Scholar in Mathematics, University of Pennsylvania.

$\ddagger$ For other papers dealing with ternary operations see A. B. Kempe, On the relation between the logical theory of classes and the geometrical theory of points, Proceedings of the London Mathematical Society, (1), vol. 21 (1890), pp. 147182; Orrin Frink, The operations of Boolean algebras, Annals of Mathematics, (2), vol. 27 (1925-1926), pp. 477-490; see also the bibliography at the end of Frink's paper.

$\S$ See the Transactions of this Society, vol. 5 (1904), pp. 288-309. 
Postulate $\mathrm{A}_{3} .(a b c)=(b c a)$.

DEFINITION 1. $a^{\prime}=(a a a)$.

Postulate $\mathrm{A}_{4} \cdot\left(a^{\prime} b b^{\prime}\right)=a$.

Postulate $\mathrm{A}_{5} \cdot{ }^{*}\left[a b(c d e)^{\prime}\right]=\left[(a b c)^{\prime}(a b d)^{\prime} e\right]$.

3. Theorems. We now list a number of theorems of the resulting algebra.

1. $a^{\prime \prime}=a$, where $a^{\prime \prime}=\left(a^{\prime}\right)^{\prime}$.

2. $(a a b)=a^{\prime}$.

3. $(a b c)=(a c b)$.

4. $(a b c)=(c b a)=(b c a)=(a c b)=(c a b)=(b a c)$.

5. $\left[a^{\prime}(a b c)^{\prime}\left(a^{\prime} b^{\prime} c^{\prime}\right)^{\prime}\right]=a$.

6. $\left[a(a b c)^{\prime}\left(a b^{\prime} c^{\prime}\right)^{\prime}\right]=a^{\prime}$.

7. $(a b c)=\left[(a b d)^{\prime}\left(a b d^{\prime}\right)^{\prime} c\right]$.

8. $\left[d^{\prime}(a b c)^{\prime}\left(a^{\prime} b^{\prime} c^{\prime}\right)^{\prime}\right]=d$.

9. If $\left(a^{\prime} b c\right)=a$ for all $a$, then $c=b^{\prime}$.

10. $(a b c)^{\prime}=\left(a^{\prime} b^{\prime} c^{\prime}\right)$.

4. Proofs of the Preceding Theorems.

Proof of 1. $a^{\prime \prime}=\left(a^{\prime \prime \prime} a^{\prime} a^{\prime \prime}\right)=\left(a^{\prime} a^{\prime \prime} a^{\prime \prime \prime}\right)=a$, by $\mathrm{A}_{4}, \mathrm{~A}_{3}, \mathrm{~A}_{4}$.

Proof of 2. $(a a b)=\left[\left(a b b^{\prime}\right)^{\prime}\left(a b b^{\prime}\right)^{\prime} b\right]=\left[a b\left(b^{\prime} b^{\prime} b\right)^{\prime}\right]=\left(a b b^{\prime}\right)$ $=a^{\prime}$, by $\mathrm{A}_{4}, 1, \mathrm{~A}_{5}, \mathrm{~A}_{4}, 1, \mathrm{~A}_{4}, 1$.

Froof of $3 . \dagger$ Put $(a c b)^{\prime}=d,\left[c(a b c)^{\prime} b\right]^{\prime}=e$. Then $(a b c)=\left[a b(c c d)^{\prime}\right]=\left[a b(c d c)^{\prime}\right]=\left[(a b c)^{\prime}(a b d)^{\prime} c\right]=\left[c(a b c)^{\prime}(a b d)^{\prime}\right]$ $=\left\{\left[c(a b c)^{\prime} a\right]^{\prime}\left[c(a b c)^{\prime} b\right]^{\prime} d\right\}=\left\{\left[c(a b c)^{\prime} a\right]^{\prime} e d\right\}=\left\{\left[a c(a b c)^{\prime}\right]^{\prime} e d\right\}$ $=\left\{\left[(a c a)^{\prime}(a c b)^{\prime} c\right]^{\prime} e d\right\}=\left\{\left[(a a c)^{\prime}(a c b)^{\prime} c\right]^{\prime} e d\right\}=\left\{\left[a(a c b)^{\prime} c\right]^{\prime} e d\right\}$ $=\left\{\left[c a(a c b)^{\prime}\right]^{\prime} e d\right\}=\left\{\left[(c a a)^{\prime}(c a c)^{\prime} b\right]^{\prime} e d\right\}=\left\{\left[(a a c)^{\prime}(c a c)^{\prime} b\right]^{\prime} e d\right\}$ $=\left\{\left[a(c a c)^{\prime} b\right]^{\prime} e d\right\}=\left\{\left[a(c c a)^{\prime} b\right]^{\prime} e d\right\}=\left[(a c b)^{\prime} e d\right]=\left[d(a c b)^{\prime} e\right]$ $=\left\{(a c b)^{\prime}(a c b)^{\prime}\left[c(a b c)^{\prime} b\right]^{\prime}\right\}=(a c b)$, by 2, 1, $\mathrm{A}_{3}, \mathrm{~A}_{5}, \mathrm{~A}_{3}, \mathrm{~A}_{5}, \mathrm{~A}_{3}$, $A_{5}, A_{3}, 2,1, A_{3}, A_{5}, A_{3}, 2,1, A_{3}, 2,1, A_{3}, 2,1$.

Proof of 4. By $\mathrm{A}_{3}, 3$.

* We shall use the symbols ( ), [ ], \{ $\}$ interchangeably to denote the fundamental ternary operation.

$\dagger \mathrm{I}$ am indebted to H. S. Zuckerman for this proof. 
In the rest of the proofs implicit use will be made of 1 and 4 .

Proof of 5. $\left[a^{\prime}(a b c)^{\prime}\left(a^{\prime} b^{\prime} c^{\prime}\right)^{\prime}\right]=\left\{\left[a^{\prime}(a b c)^{\prime} a^{\prime}\right]^{\prime}\left[a^{\prime}(a b c)^{\prime} b^{\prime}\right]^{\prime} c^{\prime}\right\}$ $=\left\{a^{\prime}\left[a^{\prime} b^{\prime}(a b c)^{\prime}\right]^{\prime} c^{\prime}\right\}=\left\{a^{\prime}\left[\left(a^{\prime} b^{\prime} a\right)^{\prime}\left(a^{\prime} b^{\prime} b\right)^{\prime} c\right]^{\prime} c^{\prime}\right\}=\left[a^{\prime} c^{\prime}\left(b^{\prime} a^{\prime} c\right)^{\prime}\right]$ $=\left[\left(a^{\prime} c^{\prime} a^{\prime}\right)^{\prime}\left(a^{\prime} c^{\prime} c\right)^{\prime} b^{\prime}\right]=\left[a^{\prime}\left(a^{\prime} c^{\prime} c\right)^{\prime} b^{\prime}\right]=\left(a^{\prime} a^{\prime} b^{\prime}\right)=a$, by $\mathrm{A}_{5}, 2$, $\mathrm{A}_{5}, \mathrm{~A}_{4}, \mathrm{~A}_{5}, 2, \mathrm{~A}_{4}, 2$.

Proof OF 6. $\left[a(a b c)^{\prime}\left(a b^{\prime} c^{\prime}\right)^{\prime}\right]=\left\{\left[a(a b c)^{\prime} a\right]^{\prime}\left[a(a b c)^{\prime} b^{\prime}\right]^{\prime} c^{\prime}\right\}$ $=\left\{a\left[a b^{\prime}(a b c)^{\prime}\right]^{\prime} c^{\prime}\right\}=\left\{a c^{\prime}\left[\left(a b^{\prime} a\right)^{\prime}\left(a b^{\prime} b\right)^{\prime} c\right]^{\prime}\right\}=\left\{a c^{\prime}\left[a\left(a b^{\prime} b\right)^{\prime} c\right]^{\prime}\right\}$ $=\left[a c^{\prime}(a a c)^{\prime}\right]=\left(a c^{\prime} a\right)=a^{\prime}$, by $\mathrm{A}_{5}, 2, \mathrm{~A}_{5}, 2, \mathrm{~A}_{4}, 2,2$.

Proof of 7. $(a b c)=\left[a b\left(d d^{\prime} c\right)^{\prime}\right]=\left[(a b d)^{\prime}\left(a b d^{\prime}\right)^{\prime} c\right]$, by $\mathrm{A}_{4}, \mathrm{~A}_{5}$.

Proof of 8. Put $(a b d)^{\prime}=p,\left(a b d^{\prime}\right)^{\prime}=q,\left(a^{\prime} b^{\prime} d\right)^{\prime}=r,\left(a^{\prime} b^{\prime} d^{\prime}\right)^{\prime}=s$. Then

$$
\begin{aligned}
{\left[d^{\prime}(a b c)^{\prime}\left(a^{\prime} b^{\prime} c^{\prime}\right)^{\prime}\right] } & =\left[d^{\prime}(p q c)^{\prime}\left(r s c^{\prime}\right)^{\prime}\right]=\left\{\left[d^{\prime} r(p q c)^{\prime}\right]^{\prime}\left[d^{\prime} s(p q c)^{\prime}\right]^{\prime} c^{\prime}\right\} \\
& =\left\{\left[\left(d^{\prime} r p\right)^{\prime}\left(d^{\prime} r q\right)^{\prime} c\right]^{\prime}\left[\left(d^{\prime} s p\right)^{\prime}\left(d^{\prime} s q\right)^{\prime} c\right]^{\prime} c^{\prime}\right\},
\end{aligned}
$$

by $7, \mathrm{~A}_{5}, \mathrm{~A}_{5}$. But $\left(d^{\prime} r q\right)=d,\left(d^{\prime} s p\right)=d$ by 5 , and $\left(d^{\prime} s q\right)=d$ by 6 . Hence the last expression reduces to

$$
\begin{aligned}
& \left\{\left[c d^{\prime}\left(d^{\prime} r p\right)^{\prime}\right]^{\prime}\left(c d^{\prime} d^{\prime}\right)^{\prime} c^{\prime}\right\}=\left\{c^{\prime} d^{\prime}\left[c d^{\prime}\left(d^{\prime} r p\right)^{\prime}\right]^{\prime}\right\}=\left[\left(c^{\prime} d^{\prime} c\right)^{\prime}\left(c^{\prime} d^{\prime} d^{\prime}\right)^{\prime}\left(d^{\prime} r p\right)^{\prime}\right] \\
& =\left[d^{\prime}\left(c^{\prime} d^{\prime} d^{\prime}\right)^{\prime}\left(d^{\prime} r p\right)^{\prime}\right]=\left[d^{\prime} d^{\prime}\left(d^{\prime} r p\right)^{\prime}\right]=d, \text { by } 2, \mathrm{~A}_{5}, \mathrm{~A}_{4}, 2,2 .
\end{aligned}
$$

Proof of 9. Suppose that for two fixed elements $b, c,\left(a^{\prime} b c\right)=a$ for every element $a$. Then for $a=c, c=\left(c^{\prime} b c\right)=b^{\prime}$ by $\mathrm{A}_{4}$.

Proof of 10. By 8, 9.

5. Sufficiency and Necessariness of the Postulates. The Whitehead-Huntington postulates leave undefined a class $K$ and two binary operations + and $X$, and are the propositions Ia, Ib, . . , VI below. In postulates IIIa-IVb the condition if the elements involved and their indicated combinations belong to $K$ is understood; in $\mathrm{V}$ the condition if the elements $Z$ and $u$ of $\mathrm{IIa}$ and $\mathrm{IIb}$ exist and are unique is understood.

Postulate Ia. $a+b$ is in $K$ whenever $a$ and $b$ are in $K$.

Postulate Ib. $a b$ is in $K$ whenever $a$ and $b$ are in $K$.

Postulate IIa. There is an element $Z$ such that $a+Z=a$ for every element $a$. 
Postulate IIb. There is an element $u$ such that $a u=a$ for every element $a$.

Postulate IIIa. $a+b=b+a$.

Postulate IIIb. $a b=b a$.

Postulate IVa. $a+b c=(a+b)(a+c)$.

Postulate IVb. $a(b+c)=a b+a c$.

Postulate V. For every element a there is an element $\bar{a}$ such that $a+\bar{a}=u$ and $a \bar{a}=Z$.

Postulate VI. There are at least two elements, $a$ and $b$, in $K$ such that $a \neq b$.

We deduce Ia-VI from our postulates as follows:

Let $u$ be any element in $K$. Then we may make the following definitions:

Definition 2. $Z=u^{\prime}$.

Definition 3. $a+b=(a b u)^{\prime}$.

DeFinition 4. $a b=(a b Z)^{\prime}$.

Proof of Ia. By Definition 3, $\mathrm{A}_{2}$, Definition 1.

Proof of Ib. By Definition 4, $\mathrm{A}_{2}$, Definition 1.

Proof of IIa. $a+Z=(a Z u)^{\prime}=\left(a u^{\prime} u\right)^{\prime}=a$, by Definition 3, Definition 2, $\mathrm{A}_{4}$.

Proof of IIb. $a u=(a u Z)^{\prime}=\left(a u u^{\prime}\right)^{\prime}=a$, by Definition 4, Definition $2, \mathrm{~A}_{4}$.

Proof of IIIa. $a+b=(a b u)^{\prime}=(b a u)^{\prime}=b+a$, by Definition 3 .

Proof of IIIb. $a b=(a b Z)^{\prime}=(b a Z)^{\prime}=b a$, by Definition 4 .

Proof of IVa. $a+b c=\left[a(b c Z)^{\prime} u\right]^{\prime}=\left[(a b u)^{\prime}(a c u)^{\prime} Z\right]^{\prime}$ $=(a+b)(a+c)$, by Definition 3, Definition 4, $\mathrm{A}_{5}$, Definition 3, Definition 4.

Proof of IVb. $a(b+c)=\left[a(b c u)^{\prime} Z\right]^{\prime}=\left[(a b Z)^{\prime}(a c Z)^{\prime} u\right]^{\prime}$ $=a b+a c$, by Definition 3, Definition 4, $\mathrm{A}_{5}$, Definition 3, Definition 4 , 
Proof of V. $a+a^{\prime}=\left(a a^{\prime} u\right)^{\prime}=u$, by Definition 3, $\mathrm{A}_{4}$. $a a^{\prime}=\left(a a^{\prime} Z\right)^{\prime}=Z$, by Definition 4, $\mathrm{A}_{4}$. Hence $a^{\prime}=\vec{a}$.

Proof of VI. By $A_{1}$.

In the converse derivation we may of course assume all the theorems of Boolean algebra, since they follow from the Whitehead-Huntington postulates. Postulates $A_{1}-A_{5}$ may then be verified without any difficulty after defining $(a b c)$ by $a^{\prime} b^{\prime}+b^{\prime} c^{\prime}+c^{\prime} a^{\prime}$.

6. Relation between Ternary and Binary Boolean Algebra. Derivation of DeMorgan's Formula. We first prove the fundamental relation $(a b c)=a^{\prime} b^{\prime}+b^{\prime} c^{\prime}+c^{\prime} a^{\prime}$.

Proof. $(a b c)=\left(a^{\prime} b^{\prime} c^{\prime}\right)^{\prime}=\left[\left(a^{\prime} b^{\prime} Z\right)^{\prime}\left(a^{\prime} b^{\prime} Z^{\prime}\right)^{\prime} c^{\prime}\right]^{\prime}$

$=\left\{\left[Z^{\prime}\left(a^{\prime} b^{\prime} Z\right)^{\prime} Z\right]^{\prime}\left(a^{\prime} b^{\prime} Z^{\prime}\right)^{\prime} c^{\prime}\right\}^{\prime}=\left\{\left[Z^{\prime}\left(a^{\prime} b^{\prime} Z\right)^{\prime} Z\right]^{\prime}\left[a^{\prime} b^{\prime}\left(Z Z^{\prime} Z^{\prime}\right)^{\prime}\right]^{\prime} c^{\prime}\right\}^{\prime}$

$=\left\{\left[Z^{\prime}\left(a^{\prime} b^{\prime} Z\right)^{\prime} Z\right]^{\prime}\left[Z^{\prime}\left(a^{\prime} b^{\prime} Z\right)^{\prime}\left(a^{\prime} b^{\prime} Z^{\prime}\right)^{\prime}\right]^{\prime} c^{\prime}\right\}^{\prime}$

$=\left\{Z^{\prime}\left(a^{\prime} b^{\prime} Z\right)^{\prime}\left[Z\left(a^{\prime} b^{\prime} Z^{\prime}\right)^{\prime} c^{\prime}\right]^{\prime}\right\}^{\prime}=\left\{Z^{\prime}\left(a^{\prime} b^{\prime} Z\right)^{\prime}\left[\left(Z c^{\prime} a^{\prime}\right)^{\prime}\left(Z c^{\prime} b^{\prime}\right)^{\prime} Z^{\prime}\right]^{\prime}\right\}^{\prime}$ $=\left\{\left(a^{\prime} b^{\prime} Z\right)^{\prime}\left[\left(b^{\prime} c^{\prime} Z\right)^{\prime}\left(c^{\prime} a^{\prime} Z\right)^{\prime} u\right]^{\prime} u\right\}^{\prime}=a^{\prime} b^{\prime}+b^{\prime} c^{\prime}+c^{\prime} a^{\prime}$, by 10, 7, $\mathrm{A}_{4}$, 2, $A_{5}, A_{5}, A_{5}$, and Definitions 2,3 , and 4 .

We next observe that 10 is a generalization of DeMorgan's formula. Indeed,

$$
(a+b)^{\prime}=(a b u)=\left(a^{\prime} b^{\prime} u^{\prime}\right)^{\prime}=\left(a^{\prime} b^{\prime} Z\right)^{\prime}=a^{\prime} b^{\prime} .
$$

7. Consistency and Independence of the Postulates.

The consistency of postulates $A_{1}-A_{5}$ is shown by the following example.

EXAMPLE 1.0. $K=1,2$.

\begin{tabular}{c|c|c|} 
& 1 & 2 \\
\hline 1 & 2 & 2 \\
\hline 2 & 2 & 1 \\
\hline
\end{tabular}

\begin{tabular}{c|c|c|} 
& 1 & 2 \\
\hline 1 & 2 & 1 \\
\hline 2 & 1 & 1 \\
\hline
\end{tabular}

The independence proofs follow.

Example 1.1. $K=1$.

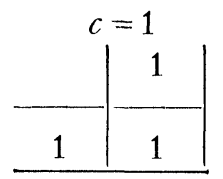


ExAmple 1.2. $K=1,2$.

\begin{tabular}{c|c|c|} 
& 1 & 2 \\
\hline 1 & 3 & 3 \\
\hline 2 & 3 & 3 \\
\hline
\end{tabular}

\begin{tabular}{c|c|c|} 
& 1 & 2 \\
\hline 1 & 3 & 3 \\
\hline 2 & 3 & 3 \\
\hline
\end{tabular}

EXAmple 1.3. $K=1,2$.

\begin{tabular}{c|c|c|} 
& 1 & 2 \\
\hline 1 & 2 & 2 \\
\hline 2 & 1 & 1 \\
\hline
\end{tabular}

\begin{tabular}{c|c|c|} 
& 1 & 2 \\
\hline 1 & 2 & 1 \\
\hline 2 & 1 & 1 \\
\hline
\end{tabular}

Postulate $\mathrm{A}_{3}$ fails for $a=2, b=c=1$.

EXAMPLE 1.4. $K=1,2$.

\begin{tabular}{c|c|c|} 
& 1 & 2 \\
\hline 1 & 1 & 1 \\
\hline 2 & 1 & 1 \\
\hline
\end{tabular}

\begin{tabular}{c|c|c|} 
& 1 & 2 \\
\hline 1 & 1 & 1 \\
\hline 2 & 1 & 1 \\
\hline
\end{tabular}

Postulate $\mathrm{A}_{4}$ fails for $a=2, b=c=1$.

ExAmple 1.5* $K=1,2$.

\begin{tabular}{c|c|c|} 
& 1 & 2 \\
\hline 1 & 1 & 2 \\
\hline 2 & 2 & 1 \\
\hline
\end{tabular}

\begin{tabular}{c|c|c|} 
& 1 & 2 \\
\hline 1 & 2 & 1 \\
\hline 2 & 1 & 2 \\
\hline
\end{tabular}

Postulate $\mathrm{A}_{5}$ fails for $a=c=1, b=d=e=2$.

The University of Pennsylvania

* The following alternate proof-systems, due in part to J. C. C. McKinsey, are of interest. Ex. 1.0. $K=1,2 ;(a b c)=[(a+b+c)(a+b+c+1) / 2](\bmod 2)$. Ex. 1.1. $K=1 ;(a b c)=1$. Ex. 1.2. $K=1,2 ;(a b c)=3$. Ex. 1.3. $K=1,2 ;(a b c)=a$. Ex. 1.4. $K=1,2 ;(a b c)=1$. Ex. 1.5. $K=1,2 ;(a b c)=[a+b+c](\bmod 2) \cdot A_{5}$ fails for $a=c=d=e=1, b=2$. 